
\title{
R Reserach S Surare \\ Thromboprophylaxis, Delta SOFA and Main Outcomes in Ventilated Patients: An Analysis of the MIMIC-III Clinical Database
}

Mrigendra M. Bastola ( $\square$ mrigendra.bastola@nih.gov)

$\mathrm{NIH} / \mathrm{NLM} / \mathrm{LHC}$

Craig Locatis

$\mathrm{NIH} / \mathrm{NLM} / \mathrm{LHC}$

Paul Fontelo

$\mathrm{NIH} / \mathrm{NLM} / \mathrm{LHC}$

\section{Research Article}

Keywords: Thrombosis, Ventilated Patients, Thromboprophylaxis

Posted Date: July 16th, 2020

DOI: https://doi.org/10.21203/rs.3.rs-43372/v1

License: (1) (i) This work is licensed under a Creative Commons Attribution 4.0 International License.

Read Full License 


\section{Abstract}

Introduction: Critical care patients are at higher risk for thromboembolic disorders. There are limited studies regarding the effect of Heparin, Warfarin, Enoxaparin and Aspirin on ventilated patients, who are likely to both benefit from prophylaxis and suffer from adverse effects of blood thinners.

Methods: This study analyzed the MIMIC-III clinical database on 4192 ventilated patients using Statistical Analysis Software (SAS, Version 9.4). Relevant data was systematically analyzed on the thromboprophylaxis agents and their effects on major treatment outcomes. Parameters studied were the length of ventilation, length of Intensive Care Unit (ICU) stays, ICU mortality, inpatient mortality, improvement in SOFA score, and occurrence of major thromboembolic events such as pulmonary embolism (PE) and deep venous thrombosis (DVT).

Results: Except Aspirin, all thromboprophylactic agents showed statistically significant reduction on ICU mortality. None of the blood thinners showed statistically significant reduction in occurrences of pulmonary embolism and deep venous thrombosis. Heparin, Warfarin and Enoxaparin had adjusted Odds ratios of $0.59(p<0.01,0.47-0.77), 0.23(p<0.05,0.1-0.57)$ and $0.36(P<0.05,0.16-0.83)$ for ICU mortality. Heparin, Warfarin and Enoxaparin had adjusted Odds ratios of $0.51(p<0.01,0.38-0.68), 0.19(p<0.01,0.06-$ $0.59)$ and $0.42(P=0.06,0.17-1.05)$ for overall ventilated patient hospital mortality, including after transfers to the inpatient ward.. Only Heparin ( $P<0.05$, OR 1.52(1.07-2.15)) was associated with thrombocytopenia episodes, which required platelets transfusion. None of the drugs showed statistically significant relationships with development of thromboembolic events after thromboprophylaxis. Only Heparin had mild effect on improvement in sequential organ failure assessment (delta SOFA) scores at 7 and 10 day after ICU admission ( $P<0.05$, OR 1.17 (1.03-1.32)).

Conclusion: Although the results supported the use of thromboprophylaxis in ventilated patients to improve treatment outcomes and decrease thromboembolic events, no benefits were indicated for using newer blood thinners (Enoxaparin) than older ones (Heparin \& Warfarin). The results of this analysis might help practitioners select, maintain, and switch blood thinners in ventilated patients.

\section{Introduction}

Thromboprophylaxis drugs are commonly used in critical care settings. Studies have shown that the rate of venous thromboembolism rises from 10 to 80 percent in ICU patients without thromboprophylaxis [1]. ICU patients have a higher risk for thromboembolic events due to immobilization and invasive procedures (intubation, central arterial and venous lines) [1,2]. Pulmonary embolism is a leading cause of death in critically ill patients [3]. Many studies advocate thromboprophylaxis for patients who are at risk of developing of thromboembolism [4]. Some studies show that newer blood thinners are better than older, due to lower incidence of adverse effects, such as thrombocytopenia related internal bleeding [5]. Practitioners routinely use coagulation tests (e.g. prothrombin time, activated partial thromboplastin time, 
international normalized ratio) and platelet counts while monitoring thromboprophylaxis agents. Although coagulation tests guide practitioners to titrate the dose of prophylactic blood thinners or switch to others, these monitoring methods give little insight regarding patient's course of care or outcomes during ventilation and ICU-related stays.

Due to the many complexities of patient management and ventilation risks, the treatment decisions for ventilated patients are more likely to be influenced by significant ICU-related outcomes, including decisions for thromboprophylaxis. Although studies support the active use of blood thinners in an ICU settings, none are considered entirely safe for ventilated patients, because of increased risks of thrombocytopenia, numerous drug interactions, and major gastrointestinal or other life-threatening bleeding episodes, requiring precise dosing and monitoring [6,7]. These life-threatening episodes can contribute towards longer ICU stay, bleeding complications, and hospital mortality. Very few publications

are available about ICU patients and the safety of thromboprophylaxis that emphasizing risks of serious complications. Protocols avoiding traditional thromboprophylaxis such as Heparin and using citrate anticoagulation rather than aiming to reduce bleeding risks have been advocated [8]. Despite thromboprophylaxis, ventilated patients seem to be at higher risk of pulmonary embolism and deep vein thrombosis, and there is lack of data on diagnosis and subsequent management $[9,10]$.

This study analyzed the effect of the thromboprophylaxis agents on ventilated patient-related outcomes, clinical improvement during ICU stay, and the effectiveness on decreasing major thromboembolic disorders (i.e., Pulmonary Embolism (PE) and Deep Venous Thrombosis (DVT)) and assessed their effects on clinical thrombocytopenia requiring platelets transfusion.

\section{Methods}

Data for this study were obtained from the publicly available MIMIC-III (Medical Information Mart for Intensive Care III) clinical database containing de-identified data from critical care patients. SQL queries in Python were used to identify patients who were ventilated and received thromboprophylaxis agents by using ICD-9 codes and generic prescription drug names. Queries included chart events, ICU stays, ICU admissions and prescriptions sections of the MIMIC-III clinical database. All ventilated patients were identified and entries with missing data were excluded.

The blood thinners studied were, Heparin, Warfarin, Enoxaparin and Aspirin. These medications comprised more than $95 \%$ of all blood thinners used in the ventilated ICU patients in the study population. Other thinners, such as Clopidogrel, Fondaparinux and Dipyridamole-Aspirin combination, did not have enough patients to include in the analysis. The authors also excluded Heparin if it was used only as a flushing agent for central or peripheral lines and included patients where its use was of therapeutic significance. Patients who had ICU stay less than 24 hours were excluded in the study since they were considered outliers.

Statistical Analysis Software (SAS, version 9.4) was used for data analysis. Regression analysis of survival data based on proportional hazards models was generated for ICU mortality indicators. Survival 
probability plots were generated for ventilated patients. Odds ratios were calculated for the thromboprophylactic agents and the occurrence of pulmonary embolism and deep venous thrombosis. Clinical outcomes reviewed were: length of ventilation, length of ICU stays, ICU mortality, inpatient mortality, risk of major internal bleeding, and occurrence of major thromboembolic events such as, pulmonary embolism and deep venous thrombosis. The Sequential Organ Failure Assessment (SOFA) score is a widely used and most accepted form of mortality prediction tool [11]. Clinical improvement was assessed using a Delta SOFA score, which is the difference between the SOFA scores at admission and SOFA scores at the $4^{\text {th }}$ (average ICU stay days), $7^{\text {th }}$, and $10^{\text {th }}$ day after ICU admission [11]. The effect of the blood thinners on Delta SOFA scores was assessed with the proportional hazard ratios and adjusted Odds ratios using logistic regression. A cumulative hazard ratio for SOFA scores at ICU admission and for length of ventilation was calculated. Logistic regression with confounders adjustment was performed and adjusted odds ratios were derived for ICU mortality, overall hospital mortality and Delta SOFA score.

\section{Results}

The median ventilation duration was less than a day ( 23.5 hours) in 4192 ventilated patients. The study population had a median age of 59 years, 2404 were males and 1788 were females. The median ICU length of stay was 2.84 days while the median hospital stay was 7.48 days. 746 subjects expired in the hospital wards and 588 expired in the ICU. The median survival duration was 40.2 days for all expired patients, and 2.45 days for those who expired during ICU stay. Other relevant characteristics are listed in Table 1.

\section{Thromboprophylaxis use and Survival plot for ventilated patients}

Of the 4192 ventilated patients, 111 patients were diagnosed with DVT and related conditions, and 70 were recorded as pulmonary embolism related categories in ICD-9. Congestive heart failure was present in 296 ventilated patients, atrial fibrillation in 669, coronary artery disease in 422, renal disorders in 280 , liver diseases in 302 and 42 had Chronic Obstructive Pulmonary Disease. 829 had malignancies, 1518 had respiratory failure, one patient had endocarditis, 68 patients had Acute Respiratory Distress Syndrome, and 650 had pneumonia. Among those who received either oral or parenteral thromboprophylaxis, 2408 of the ventilated patients received Heparin or its derivatives. 250 patients received Warfarin; 220 patients received Enoxaparin while 653 patients received Aspirin. Platelets transfusions were given to 157 patients. Clopidogrel was given to 93 patients, while four patients received Fondaparinux. Clopidogrel, Fondaparinux and Dipyridamole-Aspirin combination were excluded from further analysis due to the small sample size. 61.5 percentage $(n=2580$ patients) of the ventilated patients received Heparin, Warfarin and Enoxaparin, either alone or in combination. Among the 4192 patients, 225 were tested for D-dimer levels, 25 of them showed D-dimer levels within thenormal range ( $<500$ units). D-dimers levels rapidly increase to higher levels (in thousands) in patients with thromboembolism. Thirty patients had D-dimer levels of 1000-2000 units, while in 170 patients, the levels were more than 2000 units. 
SOFA scores at ICU admission and the duration of ventilation were positively associated with cumulative hazard (Figure 1). The survival probability curve showed sharp decreases in the first week and continued decreasing for 10 days of ICU stay, then remained low afterwards (Figure $1 \mathrm{a}$ ). Based on the survival curves (Figure 1 a \&b), SOFA scores were calculated at the 4th, 7th and 10th day of admission, and changes in the SOFA scores were analyzed relative to use of thromboprophylaxis. The effect of the blood thinners on SOFA score changes (delta SOFA) were also analyzed (Table 4). Cumulative plots showed mortality rates rising stepwise with SOFA scores at ICU admission (Figure 2). Predicted cumulative probabilities showed higher SOFA scores having higher predictive values for overall hospital mortality (Figure 2). DVTs were more likely treated with Warfarin (OR 4.19, 2.52-6.97) while pulmonary embolism patients weremore likely to receive both Warfarin (OR 5.06, 2.75-9.3) and Enoxaparin (OR 3.8, 2-7.14) during the hospital stay (Table 2).

\section{Hazard ratios for survival durations, ventilator durations and thromboembolic events}

Aspirin was not significant in any of the outcomes calculated, except for the reduction of ICU stay duration (Table 2, $3 \& 4$ ). Hazard ratio calculations included all ICU stay expired, in-patient stay expired, and discharged patients using regression analysis of data using proportional hazards model. For ICU length of stay, Heparin, Warfarin, Enoxaparin and Aspirin had hazard ratios of $0.59(p<0.01), 0.81(p<0.01)$, $0.65(p<0.01)$ and $0.88(p<0.01)$ respectively. For hospital length of stay, Heparin, Warfarin, Enoxaparin and Aspirin had hazard ratios of $0.7(p<0.01), 0.82(p<0.01), 0.62(p<0.01)$ and $0.96(p=0.38)$ respectively (Table 3). For ventilation duration, Heparin, Warfarin, Enoxaparin and Aspirin had hazard ratios of 0.66 $(p<0.01), 0.87(p=0.13), 0.64(p<0.01)$ and $0.98(p=0.59)$ respectively (Table 3$)$. When data was analyzed for occurrence of major thromboembolic events for each of the blood thinners, showed statistically significant reduction in occurrences of pulmonary embolism and deep venous thrombosis, suggesting equivocal effects among Heparin, Warfarin, Enoxaparin and Aspirin (Table 3).

\section{Adjusted odds ratios for ICU mortality, overall hospital mortality, platelets transfusion events and delta SOFAs at day $4,7 \& 10$}

The analysis was adjusted for confounders age, gender, malignancy, smoking, chronic alcoholism, SOFA score at admission, night shift admissions, night shift deaths, weekend admission and weekend deaths (Table 4) [12]. Except Aspirin, all thromboprophylactic agents showed statistically significant reduction on ICU mortality (Table 4). Heparin, Warfarin and Enoxaparin had adjusted Odds ratios of 0.59 ( $p<0.01,0.47-$ $0.77), 0.23(p<0.05,0.1-0.57)$ and $0.36(P<0.05,0.16-0.83)$ for ICU mortality (Table 4). Heparin, Warfarin and Enoxaparin had adjusted Odds ratios of $0.51(p<0.01,0.38-0.68), 0.19(p<0.01,0.06-0.59)$ and $0.42(P=0.06,0.17-1.05)$ for overall ventilated patient hospital mortality, including after transfers to the inpatient ward. Only Heparin ( $P<0.05$, OR 1.52(1.07-2.15)) was associated with serious thrombocytopenia episodes, which required platelets transfusion (Table 4). Only Heparin had mild effect 
on improvement in sequential organ failure assessment (delta SOFA) scores at 7 and 10 day after ICU admission ( $\mathrm{P}<0.05$, OR 1.17 (1.03-1.32) (Table 4).

\section{Discussion}

Heparin was the most used agent, followed by Warfarin and its derivatives. Heparin, Warfarin, Enoxaparin reduced length of ICU stays and hospital stays. These findings suggest that the use of all the thromboprophylaxis agents had comparable effects in ventilated patients and had a significant positive impact on the ICU-related outcomes in the study population. Also, the newer blood thinner, Enoxaparin, had lesser effect on the outcomes, contrary to expectations, and, perhaps, had slightly lower mortality or morbidity advantages compared to the older blood thinners such as Heparin and Warfarin (Table 2 \&3). This finding contradicts the findings of a published study where Enoxaparin or Dalteparin were found at least as effective as unfractionated Heparin [13]. This study showed high use of thromboprophylaxis among ventilated patients (61.5\%), comparable with other published studies, which reported overall thromboprophylaxis at $61.8 \%$ for all indicated patients during a hospital stay [14]. Also, the occurrences of pulmonary embolism, deep venous thrombosis (DVT) during the hospital stay were statistically significant for none of the blood thinners (Table 3). These findings are comparable with those of a previous study which did not find thromboprophylaxis prevents PE $[15,16]$. Severe thrombocytopenia is a life-threatening complication, and can potentially cause internal bleeding, (e.g. intracerebral hemorrhage) if not treated. In this study, only Heparin was associated with serious thrombocytopenia episodes which required platelets transfusion (Table 4).

The number of individuals diagnosed with pulmonary embolism $(n=70)$ and DVT $(n=$ 111) was small. Moreover, the occurrence of either pulmonary embolism or DVT did not vary significantly between any of the blood thinners studied (Table 2 \&3). However, DVTs were more likely treated with Warfarin while pulmonary embolism patients weremore likely to receive both Warfarin and Enoxaparin during their hospital stay (Table 2). These findings align with the contemporary clinical practice where the individuals who developed PE and DVT during the hospital stay arepresumably switched or other thromboprophylactic agents are added. Also, higher D-dimerlevels were not significantly associated with any of the blood thinners studied, although some studies havesuggested that Heparin use might causealterations in D-dimer levels (Table 4) [17].

\section{Limitations:}

A limitation of the study is the paucity of available data and publications on newer blood thinners (e.g. Dabigatran and Fondaparinux) which had low utilization levels in the study population. A more extensive study for these and newer blood thinners might be needed. Additionally, most of the oral and newer anticoagulants were used in combination with the older medications, such as Heparin and its derivatives. This study included patients of multiple comorbidities with diverse risk factors for the development of thromboembolic events such as, immobilization, sepsis, surgery, trauma, multiple organ failures, 
malignancy, sedation, paralysis, and indwelling catheters. A comorbidity specific study of thromboprophylaxis, such as sepsis versus surgery, is desirable in future studies to analyze the choice of the anticoagulants in detail.

\section{Conclusion}

The findings of this study support the use of thromboprophylaxis with Heparin, Warfarin and Enoxaparin, either alone or in combination, to improve ICU stay outcomes by reducing the duration of hospital and ICU stays, lowering ICU mortality, and reducing ventilator duration. Although the results supported the use of thromboprophylaxis in ventilated patients to improve treatment outcomes and decrease thromboembolic events, this analysis did not show any benefits of newer blood thinners (e.g. Enoxaparin) when compared to older ones (e.g. Heparin and Warfarin) as suggested by some studies. The results of this analysis might help practitioners during the selection and switching of blood thinners in ventilated patients, if supported by further studies.

\section{List Of Abbreviations}

\begin{tabular}{|ll|}
\hline D1, D4, D7, D10 & Day 1, Day 4, Day 7, Day10. \\
\hline Delta-SOFA & Delta Sequential organ failure assessment score \\
\hline DVT & Deep Venous Thrombosis \\
\hline ICD-9 & International Classification of Diseases, Ninth Revision \\
\hline ICU & Intensive Care Unit \\
\hline LOS & Length of Stay \\
\hline MIMIC-III & Medical Information Mart for Intensive Care III \\
\hline OR & Odds Ratio \\
\hline PE & Pulmonary Embolism \\
\hline PP & Platelet rich plasma \\
\hline SOFA score & Sequential organ failure assessment score \\
\hline SQL & Structured Query Language \\
\hline
\end{tabular}

\section{Declarations}

Ethics approval and consent to participate: Not applicable.

Consent for publication: Not applicable.

Availability of data and materials: 
The data used in this review are extracted from publicly available, deidentified MIMIC-III clinical database (URL: https://physionet.org/content/mimiciii/1.4/). Additionally, the deidentified datasets used and data analysis output from SAS are available from the corresponding author upon reasonable request.

Competing interests: Authors declare no competing interests.

\section{Funding:}

This research was supported by the Intramural Research Program of the National Institutes of Health $(\mathrm{NIH})$, National Library of Medicine (NLM) and Lister Hill National Center for Biomedical Communications (LHNCBC). The funding body had no role in the design of the study, data collection, data analysis, interpretation of the findings and writing of the manuscript.

\section{Authors' contributions:}

PF, MB, and CL conceived the idea and design for the paper. MB performed the research on the MIMIC-III clinical database, including data collection and statistical analysis. MB wrote the main draft of the paper, including tables and figures. PF and CL assisted in writing the paper. All of the authors reviewed and approved the final version of the manuscript.

\section{Acknowledgements:}

The views and opinions of the authors herein do not necessarily state or reflect those of the National Library of Medicine, National Institutes of Health or the US Department of Health and Human Services.

\section{References}

1. Adriance S, Murphy C. Prophylaxis and treatment of venous thromboembolism in the critically ill. International Journal of Critical IIIness and Injury Science. 2013;3(2):143.

2. Ejaz A, Ahmed M, Tasleem A, Khan Niazi M, Ahsraf M, Ahmad I et al. Thromboprophylaxis in Intensive Care Unit Patients: A Literature Review. Cureus. 2018;.

3. Howard R. General medical care on the neuromedical intensive care unit. Journal of Neurology, Neurosurgery \& Psychiatry. 2003;74(90003):10iii-15.

4. Zhang C, Zhang Z, Mi J, Wang X, Zou Y, Chen X et al. The cumulative venous thromboembolism incidence and risk factors in intensive care patients receiving the guideline-recommended thromboprophylaxis. Medicine. 2019;98(23):e15833.

5. Toews I, George A, Peter J, Kirubakaran R, Fontes L, Ezekiel J et al. Interventions for preventing upper gastrointestinal bleeding in people admitted to intensive care units. Cochrane Database of Systematic Reviews. 2018;

6. Ia N. Outcome Analysis of Anticoagulant Therapy in Critical Care Unit: The Need for a Pharmacy Managed Anticoagulant Service. Advancements in Cardiovascular Research. 2019;2(1). 
7. Marras E, Lo Nigro L, Berlot G. Anticoagulation Therapy in ICU Patients. Hemocoagulative Problems in the Critically III Patient. 2012;:37-60.

8. Hetzel G, Schmitz M, Wissing H, Ries W, Schott G, Heering P et al. Regional citrate versus systemic heparin for anticoagulation in critically ill patients on continuous venovenous haemofiltration: a prospective randomized multicentre trial. Nephrology Dialysis Transplantation. 2010;26(1):232-239.

9. Zochios V, Keeshan A. Pulmonary Embolism in the Mechanically-Ventilated Critically III Patient: Is it Different?. Journal of the Intensive Care Society. 2013;14(1):36-44.

10. Ibrahim E, Iregui M, Prentice D, Sherman G, Kollef M, Shannon W. Deep vein thrombosis during prolonged mechanical ventilation despite prophylaxis. Critical Care Medicine. 2002;30(4):771-774.

11. García-Gigorro R, Sáez-de la Fuente I, Marín Mateos H, Andrés-Esteban E, Sanchez-Izquierdo J, Montejo-González J. Utility of SOFA and $\triangle$-SOFA scores for predicting outcome in critically ill patients from the emergency department. European Journal of Emergency Medicine. 2018;25(6):387393.

12. Orban J, Walrave Y, Mongardon N, Allaouchiche B, Argaud L, Aubrun F et al. Causes and Characteristics of Death in Intensive Care Units. Anesthesiology. 2017;126(5):882-889.

13. Norris T, Alexander T. Thromboprophylaxis in medical patients: the role of low-molecular-weight heparin. Thrombosis and Haemostasis. 2004;92(07):3-12.

14. Amin A, Stemkowski S, Lin J, Yang G. Thromboprophylaxis rates in US medical centers: success or failure?. Journal of Thrombosis and Haemostasis. 2007;5(8):1610-1616.

15. Barrera L, Perel P, Ker K, Cirocchi R, Farinella E, Morales Uribe C. Thromboprophylaxis for trauma patients. Cochrane Database of Systematic Reviews. 2013;.

16. Rausa E, Kelly M, Asti E, Aiolfi A, Bonitta G, Winter D et al. Extended versus conventional thromboprophylaxis after major abdominal and pelvic surgery: Systematic review and meta-analysis of randomized clinical trials. Surgery. 2018;164(6):1234-1240.

17. Couturaud F, Kearon C, Bates S, Ginsberg J. Decrease in sensitivity of D-dimer for acute venous thromboembolism after starting anticoagulant therapy. Blood Coagulation \& Fibrinolysis. 2002;13(3):241-246.

\section{Tables}

Due to technical limitations, Tables 1-4 are provided in the Supplementary Files section.

\section{Figures}




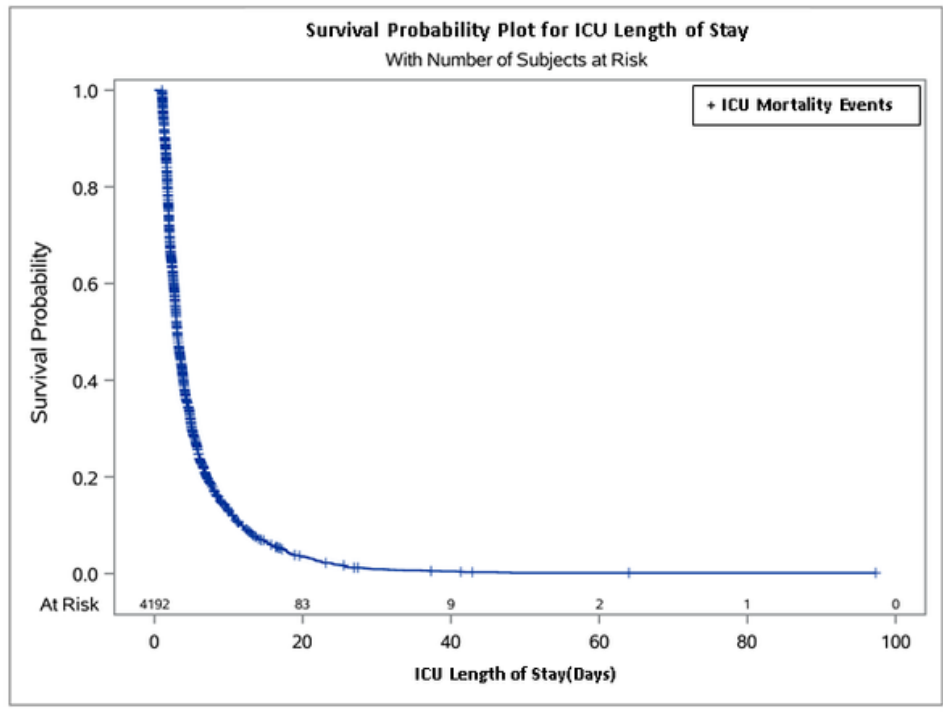

(a)

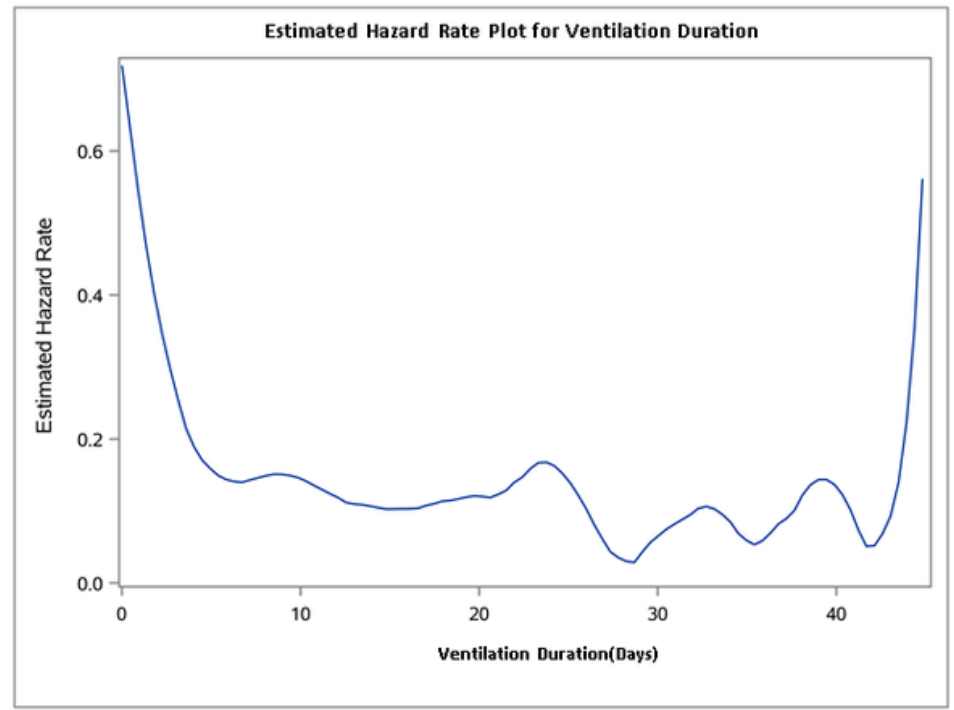

(b)

Figure 1. Survivor probability for length of ICU stay (a) and estimated hazard rate plot for ventilation duration (b).

\section{Figure 1}

Survivor probability for length of ICU stay (a) and estimated hazard rate plot for ventilation duration (b).

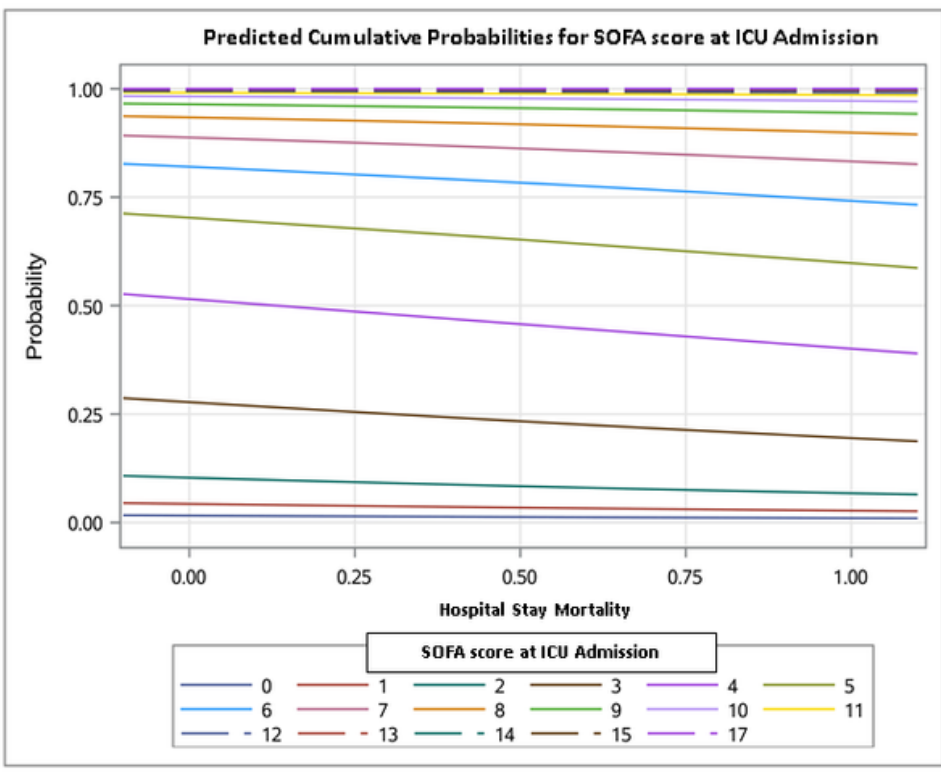

(a)

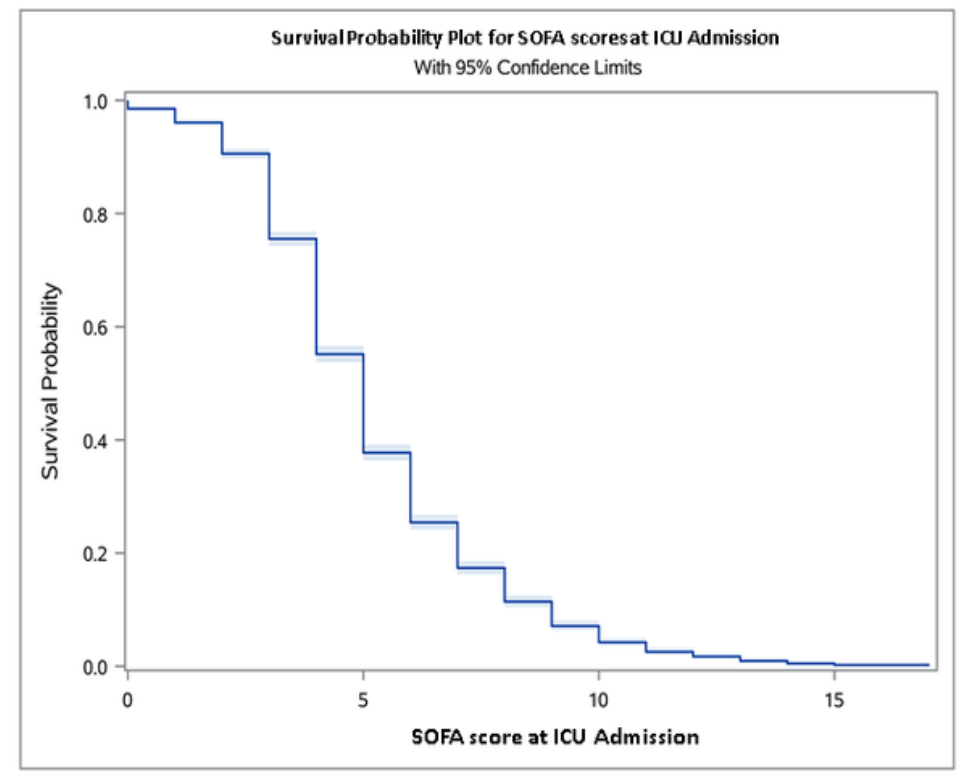

(b)

Figure 2. Predicted cumulative probabilities for SOFA scores at ICU admission. (a) SOFA scores at admission plotted against hospital mortality. The dotted lines represent high SOFA scores (12-17), and the lines below (in order, 0 lowest, 11 highest) for lower SOFA scores at ICU admission. (b)Survival plot for SOFA scores at ICU admission. 
Figure 2

Predicted cumulative probabilities for SOFA scores at ICU admission. (a) SOFA scores at admission plotted against hospital mortality. The dotted lines represent high SOFA scores (12-17), and the lines below (in order, 0 lowest, 11 highest) for lower SOFA scores at ICU admission. (b)Survival plot for SOFA scores at ICU admission.

\section{Supplementary Files}

This is a list of supplementary files associated with this preprint. Click to download.

- Table3.pptx

- Table1.pdf

- Table2.pdf

- Table4.pptx 